Character list is expanded and modified from the O'Connor et al. (2012) [9] dataset. We have removed one taxon (Zhongornis haoae), added two taxa (Eopengornis martini, and Parapengornis eurycaudatus), and three additional post-cranial characters (characters 246, 247, 248). All 248 characters from this list were included in this analysis.

\title{
Skull and Mandible
}

1. Premaxillae in adults: unfused (0); fused only rostrally (1); completely fused (2). (ORDERED)

2. Maxillary process of the premaxilla: restricted to its rostral portion (0); subequal or longer than the facial contribution of the maxilla (1).

3. Frontal process of the premaxilla: short (0); relatively long, approaching the rostral border of the antorbital fenestra (1); very long, extending caudally near the level of lacrimals (2). (ORDERED)

4. Premaxillary teeth: present throughout (0); present but rostral tip edentulous (1); present but restricted to rostral portion (2); absent (3).

5. Caudal margin of naris: far rostral than the rostral border of the antorbital fossa (0); nearly reaching or overlapping the rostral border of the antorbital fossa (1).

6. Naris longitudinal axis: considerably shorter than the long axis of the antorbital fossa (0); subequal or longer (1). We are using the longitudinal axis of these structures as a proxy for their relative size. The longitudinal axis is often easier to measure than the actual area enclosed by either the naris or the antorbital fossa. 
7. Maxillary teeth: present (0); absent (1).

8. Dorsal (ascending) ramus of the maxilla: present with two fenestra (the promaxilllary and maxillary fenestra) (0); present with one fenestra (1); unfenestrated (2); ramus absent (3). (ORDERED)

9. Caudal margin of choana: located rostrally, not overlapping the region of the orbit (0); displaced caudally, at the same level or overlapping the rostral margin of the orbit (1).

10. Rostral margin of the jugal: away from the caudal margin of the naris (0); or very close to (leveled with) the caudal margin of the naris (1).

11. Contact between palatine and maxilla/premaxilla: palatine contact maxilla only (0); contacts premaxilla and maxilla (1).

12. Vomer and pterygoid articulation: present, well developed (0); reduced, narrow process of pterygoid passes dorsally over palatine to contact vomer (1); absent, pterygoid and vomer do not contact (2).

13. Jugal process of palatine: present (0); absent (1).

14. Contact between palatine and pterygoid: long, craniocaudally overlapping contact (0); short, primarily dorsoventral contact (1).

15. Contact between vomer and premaxilla: present (0); absent (1).

16. Ectopterygoid: present (0); absent (1).

17. Postorbital: present (0); absent (1).

18. Contact between postorbital and jugal: present (0); absent (1).

19. Quadratojugal: sutured to the quadrate (0); joined through a ligamentary 
articulation (1).

20. Lateral, round cotyla on the mandibular process of the quadrate (quadratojugal articulation): absent (0); present (1).

21. Contact between the quadratojugal and squamosal: present (0); absent (1).

22. Squamosal incorporated into the braincase, forming a zygomatic process: absent (0); present (1).

23. Squamosal, ventral or "zygomatic" process: variably elongate, dorsally enclosing otic process of the quadrate and extending cranioventrally along shaft of this bone, dorsal head of quadrate not visible in lateral view (0); short, head of quadrate exposed in lateral view (1).

24. Frontal/parietal suture in adults: open (0); fused (1).

25. Quadrate orbital process (pterygoid ramus): broad (0); sharp and pointed (1).

26. Quadrate pneumaticity: absent (0); present (1).

27. Quadrate: articulating only with the squamosal (0); articulating with both prootic and squamosal (1).

28. Otic articulation of the quadrate: articulates with a single facet (squamosal) (0); articulates with two distinct facets (prootic and squamostal) (1); articulates with two distinct facets and quadrate differentiated into two heads (2). (ORDERED)

29. Quadrate distal end: with two transversely aligned condyles (0); with a triangular, condylar pattern, usually composed of three distinct condyles (1).

30. Basipterygoid processes: long (0); short (articulation with pterygoid subequal to, or longer than, amount projected from the basisphenoid rostrum) (1). 
31. Pterygoid, articular surface for basipterygoid process: concave "socket", or short groove enclosed by dorsal and ventral flanges (0); flat to convex (1); flat to convex facet, stalked, variably projected (2). (ORDERED)

32. Eustachian tubes: paired, lateral, and well-separated from each other (0); paired, close to each other and to cranial midline or forming a single cranial opening (1).

33. Osseous interorbital septum (mesethmoid): absent (0); present (1).

34. Dentary teeth: present (0); absent (1).

35. Dentary tooth implantation: teeth in individual sockets (0); teeth in a communal groove (1).

36. Symphysial portion of dentaries: unfused (0); fused (1).

37. Deeply notched rostral end of the mandibular symphysis: absent (0); present (1).

38. Mandibular symphysis, symphyseal foramina: absent (0); single (1); paired (2).

39. Mandibular symphysis, symphyseal foramen/foramina: opening on caudal edge of symphysis (0); opening on dorsal surface of symphysis (1).

40. Small ossification present at the rostral tip of the mandibular symphysis (intersymphysial ossification): absent (0); present (1). Martin refers to this ossification as the "predentary." This term is inappropriate as it implies a homology between this ossification and the predentary bone of ornithischian dinosaurs - a hypothesis that is not supported by parsimony.

41. Caudal margin of dentary strongly forked: unforked, or with a weakly developed dorsal ramus (0); strongly forked with the dorsal and ventral rami approximately equal in caudal extent (1). 
42. Mandibular ramus sigmoidal such that the rostral tip is dorsally convex and the caudal end is dorsally concave: absent (0); present (1).

43. Cranial extent of splenial: stops well caudal to mandibular symphysis (0); extending to mandibular symphysis, though noncontacting (1); extending to proximal tip of mandible, contacting on midline (2). (ORDERED)

44. Meckel's groove (medial side of mandible): not completely covered by splenial, deep and conspicuous medially (0); covered by splenial, not exposed medially (1).

45. Rostral mandibular fenestra: absent (0); present (1).

46. Caudal mandibular fenestra: present (0); absent (1). We regard the caudal mandibular fenestra of neornithines as homologous to the surangular fenestra of non-avian dinosaurs [1].

47. Articular pneumaticity: absent (0); present (1).

48. Teeth: serrated crowns (0); unserrated crowns (1).

\section{Vertebral Column and Ribs}

49. Atlantal hemiarches in adults: unfused (0); fused, forming a single arch (1).

50. One or more pneumatic foramina piercing the centra of mid-cranial cervicals, caudal to the level of the parapophysis-diapophysis: present (0); absent (1).

51. Cervical vertebrae: variably dorsoventrally compressed, amphicoelous ("biconcave": flat to concave articular surfaces) (0); cranial surface heterocoelous (i.e., mediolaterally concave, dorsoventrally convex), caudal surface flat or slightly concave (1); heterocoelous cranial (i.e., mediolaterally concave, 
dorsoventrally convex) and caudal (i.e., mediolaterally convex, dorsoventrally concave) surfaces (2). (ORDERED)

52. Prominent carotid processes in the intermediate cervicals: absent (0); present (1).

53. Postaxial cervical epipophyses: prominent, projecting further back from the postzygapophysis (0); weak, not projecting further back from the postzygapophysis, or absent (1).

54. Keel-like ventral surface of cervical centra: absent (0); present (1).

55. Prominent (50\% or more the height of the centru's cranial articular surface) ventral processes of the cervicothoracic vertebrae: absent (0); present (1).

56. Thoracic vertebral count: 13-14 (0); 11-12 (1); fewer than 11 (2). The transition between cervical and thoracic vertebrae is often difficult to identify, which makes counting these vertebrae problematic. Here, thoracic vertebrae are defined as possessing free, ventrally projecting ribs. When inarticulated, vertebral morphology should be used. (ORDERED)

57. Thoracic vertebrae: at least part of series with subround, central articular surfaces (e.g., amphicoelous/opisthocoelous) that lack the dorsoventral compression seen in heterocoelous vertebrae (0); series completely heterocoelous (1).

58. Caudal thoracic vertebrae, centra, length and midpoint width: approximately equal in length and midpoint width (0); length markedly greater than midpoint width (1).

59. Wide vertebral foramen in the mid-caudal thoracic vertebrae, vertebral foramen/articular cranial surface ratio (vertical diameter) larger than 0.40: absent 
(0); present (1).

60. Hyposphene-hypantrum accessory intervertebral articulations in the thoracic vertebrae: present (0); absent (1).

61. Lateral side of the thoracic centra: weakly or not excavated (0); deeply excavated by a groove (1); excavated by a broad fossa (2).

62. Cranial thoracic vertebrae, parapophyses: located in the cranial part of the centra of the thoracic vertebrae (0); located in the central part of the centra of the thoracic vertebrae (1).

63. Notarium: absent (0); present (1).

64. Sacral vertebrae, number ankylosed (synsacrum): less than 7 (0); 7 (1); 8 (2); 9 (3); $10(4) ; 11$ or more (5); 15 or more (6). (ORDERED)

65. Synsacrum, procoelous articulation with last thoracic centrum (deeply concave facet of synsacrum receives convex articulation of last thoracic centrum): absent (0); present (1).

66. Cranial vertebral articulation of first sacral vertebra: approximately equal in height and width (0); wider than high (1).

67. Series of short sacral vertebrae with dorsally directed parapophyses just cranial to the acetabulum: absent (0); present, three such vertebrae (1); present, four such vertebrae (2). (ORDERED)

68. Convex caudal articular surface of the synsacrum: absent (0); present (1).

69. Degree of fusion of distal caudal vertebrae: fusion absent (0); few vertebrae partially ankylosed (intervening elements are well-discernable) (1); vertebrae 
completely fused into a pygostyle (2). (ORDERED)

70. Free caudal vertebral count: more than $35(0)$; 35-26 (1); 25 - 20 (2); 19-9 (3); 8 or less (4). (ORDERED)

71. Procoelous caudals: absent (0); present (1).

72. Distal caudal vertebra prezygapophyses: elongate, exceeding the length of the centrum by more than $25 \%(0)$; shorter (1); absent (2). (ORDERED)

73. Free caudals, length of transverse processes: approximately equal to, or greater than, centrum width (0); significantly shorter than centrum width (1).

74. Proximal haemal arches: elongate, at least 3 times longer than wider (0); shorter (1); absent (2). (ORDERED)

75. Pygostyle: longer than or equal to the combined length of the free caudals $(0)$; shorter (1).

76. Cranial end of pygostyle dorsally forked: absent (0); present (1).

77. Cranial end of pygostyle with a pair of laminar, ventrally projected processes: absent (0); present (1).

78. Distal constriction of pygostyle: absent (0); present (1). In the pygostyles of some enantiornithine taxa, the distal-most mediolateral width is reduced so that the midline of the pygostyle projects distally farther than the lateral margins [1].

79. Ossified uncinate processes in adults: absent (0); present and free (1); present and fused (2).

80. Uncinate process, orientation: perpendicular to rib (0); angled dorsally defining an acute angle with the rib (1). 
81. Gastralia: present (0); absent (1).

\section{Thoracic Girdle and Sternum}

82. Coracoid shape: rectangular to trapezoidal in profile (0); strutlike (1).

83. Coracoid and scapula articulation: through a wide, sutured articulation (0); through more localized facets (1).

84. Scapula: articulated at the shoulder (proximal) end of the coracoid (0); well below it (1).

85. Coracoid, humeral articular (glenoid) facet: dorsal to acrocoracoid process/“biceps tubercle" (0); ventral to acrocoracoid process (1).

86. Humeral articular facets of the coracoid and the scapula: placed in the same plane (0); forming a sharp angle (1).

87. Coracoid, acrocoracoid: straight (0); hooked medially (1).

88. Laterally compressed shoulder end of coracoid, with nearly aligned acrocoracoid process, humeral articular surface, and scapular facet, in dorsal view: absent (0); present (1).

89. Procoracoid process on coracoid: absent (0); present (1).

90. Distinctly convex lateral margin of coracoid: absent (0); present (1).

91. Broad, deep fossa on the dorsal surface of the coracoid (dorsal coracoidal fossa): absent (0); present (1).

92. Supracoracoidal nerve foramen of coracoid: centrally located (0); displaced toward (often as an incisure) the medial margin of the coracoid (1); displaced so 
that it nerve no longer passes through the coracoid (absent) (2). (ORDERED) In some taxa the $\mathrm{n}$. supracoracoideus does not pierce the coracoid, but is assumed to pass medially at the level between the bone's midpoint and its glenoid (humeral articular facet).

93. Coracoid, medial surface, strongly depressed elongate furrow at the level of the passage of $\mathrm{n}$. supracoracoideus: absent (0); present (1).

94. Supracoracoid nerve foramen, location relative to dorsal coracoidal fossa: above fossa (0); inside fossa (1).

95. Coracoid, sternolateral corner: unexpanded (0); expanded (1); well developed squared-off lateral process (sternocoracoidal process) (2); present and with a distinct omal projection (hooked) (3).

96. Scapular shaft: straight (0); sagittally curved (1).

97. Scapula, length: shorter than humerus (0); as long as or longer than humerus (1).

98. Scapular acromion costolaterally wider than deeper: absent (0); present (1).

99. Scapula, acromion process: projected cranially surpassing the articular surface for coracoid (facies articularis coracoidea [11]) (0); projected less cranially than the articular surface for coracoid (1).

100. Scapula, acromion process: straight (0); laterally hooked tip (1).

101. Proximal end of scapula, pit between acromion and humeral articular facet (scapular fossa): absent (0); present (1).

102. Costal surface of scapular blade with prominent longitudinal furrow: absent (0); present (1). 
103. Scapular caudal end: blunt (may or may not be expanded) (0); sharply tapered (1).

104. Furcular, shape: boomerang-shaped (0); V to Y-shaped (1); U-shaped (2).

105. Furcula interclavicular angle: approximately $90^{\circ}(0)$; less than $70^{\circ}(1)$. The interclavicular angle is measured as the angle formed between three points, one at the omal end of each rami and the apex located at the clavicular symphysis.

106. Dorsal and ventral margins of the furcula: subequal in width (0); ventral margin distinctly wider than the dorsal margin so that the furcular ramus appears concave laterally (1).

107. Hypocleideum: absent (0); present as a tubercle or short process (1); present as an elongate process approximately $30 \%$ rami length (2); hypertrophied, exceeding $50 \%$ rami length (3). ORDERED

108. Sternum: unossified (0); partially ossified, coracoidal facets cartilaginous (1); fully ossified (2).

109. Ossified sternum: two flat plates (0); single flat element (1); single element, with slightly raised midline ridge (2); single element, with projected carina (3).

110. Sternal carina: near to, or projecting rostrally from, the cranial border of the sternum (0); not reaching the cranial border of the sternum (1).

111. Sternum, caudal margin, number of paired caudal trabecula: none (0); one (1); two (2). The use of "lateral" and "medial" to identify the specific sternal processes is abandoned here due to the difficulty of identifying trabecula when only one is present. Eoenantiornis is scored as a "?" due to the uncertain status of the sternal 
processes; it is possible that the identified "lateral process" [23] is actually the distal humerus.

112. Sternum, outermost trabecula, shape: tips terminate cranial to caudal end of sternum (0); tips terminate at or approaching caudal end of sternum (1); tips extend caudally past the termination of the sternal midline (2).

113. Prominent distal expansion in the outermost trabecula of the sternum: absent $(0)$; present (1).

114. Rostral margin of the sternum broad and rounded: absent (0); present (1).

115. Sternum, coracoidal sulci spacing on cranial edge: widely separated mediolaterally (0); adjacent (1); crossed on midline (2). In taxa such as Eoalulavis in which the preserved sternum does not bear actual sulci, the placement of the coracoids can be used to infer their position relative to the sternum.

116. Costal facets of the sternum: absent (0); present (1).

117. Sternal costal processes: three (0); four (1); five (2); six (3); seven (4); eight (5). (ORDERED)

118. Sternal midline, caudal end: blunt W-shape (0); V-shape (1); elongate straight projection (xiphoid process) (2); flat (3); rounded (4).

119. Sternum, caudal half, paired enclosed fenestra: absent (0); present (1).

120. Sternum, dorsal surface, pneumatic foramen (or foramina): absent (0); present (1).

\section{Thoracic Limb}


121. Proximal and distal humeral ends: twisted (0); expanded nearly in the same plane (1).

122. Humeral head: concave cranially and convex caudally (0); globe shaped, craniocaudally convex (1).

123. Proximal margin of the humeral head concave in its central portion, rising ventrally and dorsally: absent (0); present (1).

124. Humerus, proximocranial surface, well-developed circular fossa on midline: absent (0); present (1).

125. Humerus with distinct transverse ligamental groove: absent (0); present (1).

126. Humerus, ventral tubercle projected caudally, separated from humeral head by deep capital incision: absent (0); present (1).

127. Pneumatic fossa in the caudoventral corner of the proximal end of the humerus: absent or rudimentary (0); well developed (1).

128. Humerus, deltopectoral crest: projected dorsally (the plane of the crest is coplanar to the cranial surface of the humerus) (0); projected cranially (1).

129. Humerus, deltopectoral crest: less than shaft width (0); approximately same width (1); prominent and subquadrangular (i.e., subequal length and width) (2).

130. Humerus, deltopectoral crest, perforated by a large fenestra: absent (0); present (1).

131. Humerus, bicipital crest: little or no cranial projection (0); developed as a cranial projection relative to shaft surface in ventral view (1); hypertrophied, rounded tumescence (2). 
132. Humerus, distal end of bicipital crest, pit-shaped fossa for muscular attachment: absent (0); craniodistal on bicipital crest (1); directly ventrodistal at tip of bicipital crest (2); caudodistal, variably developed as a fossa (3).

133. Distal end of the humerus very compressed craniocaudally: absent (0); present (1).

134. Humerus, demarcation of muscle origins (e.g., m. extensor metacarpi radialis in Aves) on the dorsal edge of the distal humerus: no indication (0); a variably projected scar-bearing tubercle (dorsal supracondylar process) (2).

135. Well-developed brachial depression on the cranial face of the distal end of the humerus: absent (0); present (1). We interpret the brachial fossa not as a depression on the craniodistal end of the humerus but as a distinct scar for muscle attachment.

136. Well-developed olecranon fossa on the caudal face of the distal end of the humerus: absent (0); present (1).

137. Humerus, distal end, caudal surface, groove for passage of m. scapulotriceps: absent (0); present (1).

138. Humerus, m. humerotricipitalis groove: absent (0); present as a well-developed ventral depression contiguous with the olecranon fossa (1).

139. Humerus, distal margin: approximately perpendicular to long axis of humeral shaft (0); ventrodistal margin projected significantly distal to dorsodistal margin, distal margin angling strongly ventrally (sometimes described as a well-projected flexor process) (1). 
140. Humeral distal condyles: mainly located on distal aspect (0); on cranial aspect (1).

141. Humerus, long axis of dorsal condyle: at low angle to humeral axis, proximodistally oriented (0); at high angle to humeral axis, almost transversely oriented (1).

142. Humerus, distal condyles: subround, bulbous (0); weakly defined, "straplike" (1).

143. Humerus, ventral condyle: length of long axis of condyle less than the same measure of the dorsal condyle (0); same or greater (1).

144. Ulna: shorter than humerus (0); nearly equivalent to or longer than humerus (1).

145. Ulnar shaft, radial-shaft/ulnar-shaft ratio: larger than $0.70(0)$; smaller than 0.70 (1).

146. Ulna, cotylae: dorsoventrally adjacent (0); widely separated by a deep groove (1).

147. Ulna, dorsal cotyla strongly convex: absent (0); present (1).

148. Ulna, bicipital scar: absent (0); developed as a slightly raised scar (1); developed as a conspicuous tubercle (2).

149. Proximal end of the ulna with a well-defined area for the insertion of $\mathrm{m}$. brachialis anticus: absent (0); present (1).

150. Semilunate ridge on the dorsal condyle of the ulna: absent (0); present (1).

151. Shaft of radius with a long longitudinal groove on its ventrocaudal surface: absent (0); present (1). 
152. Ulnare: heart-shaped with little differentiation into short rami (0); U-shaped to V-shaped, well-developed rami (1).

153. Ulnare, ventral ramus (crus longus [11]): shorter than dorsal ramus (crus brevis) (0); same length as dorsal ramus (1); longer than dorsal ramus (2).

154. Semilunate carpal and proximal ends of metacarpals in adults: unfused (0); semilunate fused to the alular (I) metacarpal (1); semilunate fused to the major (II) and minor (III) metacarpals (2); fusion of semilunate and all metacarpals (3). Any specimen that is inferred to be a juvenile should be scored as a "?" in order to account for the possibility of ontogenetic change.

155. Semilunate carpal, position relative to the alular metacarpal (I): over entire proximal surface (0); over less than one-half proximal surface or no contact present (1).

156. Carpometacarpus, proximal ventral surface: flat (0); raised ventral projection contiguous with minor metacarpal (1); pisiform process forming a distinct peg-like projection (2).

157. Carpometacarpus, ventral surface, supratrochlear fossa deeply excavating proximal surface of pisiform process: absent (0); present (1).

158. Round-shaped alular metacarpal (I): absent (0); present (1).

159. Alular metacarpal (I), extensor process: absent, no cranioproximally projected muscular process (0); present, tip of extensor process just surpassed the distal articular facet for phalanx 1 in cranial extent (1); tip of extensor process conspicuously surpasses articular facet by approximately half the width of facet, 
producing a pronounced knob (2); tip of extensor process conspicuously surpasses articular facet by approximately the width of facet, producing a pronounced knob (3). (ORDERED)

160. Alular metacarpal (I), distal articulation with phalanx I: ginglymoid (0); shelf (1); ball-like (2).

161. Metacarpal III, craniocaudal diameter as a percentage of same dimension of metacarpal II: approximately equal or greater than 50\% (0); less than 50\% (1).

162. Proximal extension of metacarpal III: level with metacarpal II (0); ending distal to proximal surface of metacarpal II (1).

163. Intermetacarpal process or tubercle on metacarpal II: absent (0); present as scar (1); present as tubercle or flange (2).

164. Intermetacarpal space: absent or very narrow (0); at least as wide as the maximum width of minor metacarpal (III) shaft (1).

165. Intermetacarpal space: reaches proximally as far as the distal end of metacarpal I (0); terminates distal to end of metacarpal I (1).

166. Distal end of metacarpals: unfused (0); partially or completely fused (1).

167. Minor metacarpal (III) projecting distally more than the major metacarpal (II): absent (0); present (1).

168. Alular digit (I): long, exceeding the distal end of the major metacarpal (0); subequal (1); short, not surpassing this metacarpal (2). (ORDERED)

169. Proximal phalanx of major digit (II): of normal shape (0); flat and craniocaudally expanded (1). 
170. Major digit (II), phalanx 1, "internal index process” (Stegmann, 1978) on caudodistal edge: absent (0); present (1).

171. Second phalanx of major digit (II): longer than proximal phalanx (0); shorter than or equivalent to proximal phalanx (1).

172. Ungual phalanx of major digit (II): present (0); absent (1).

173. Ungual phalanx of major digit (II) much smaller than the unguals of the alular (I) and minor (III) digits: absent (0); present (1).

174. Proximal phalanx of the minor digit (III) much shorter than the remaining non-ungual phalanges of this digit: absent (0); present (1).

175. Ungual phalanx of minor digit (III): present (0); absent (1).

176. Length of manus (semilunate carpal + major metacarpal and digit) relative to humerus: longer (0); subequal (1); shorter (2). (ORDERED)

177. Intermembral index $=($ length of humerus + ulna $) /($ length of femur + tibiotarsus $)$ : less than 0.7, flightless (0); between 0.7 and 0.9 (1); between 0.9 and 1.1 (2); greater than $1.1(3)$.

\section{Pelvic Girdle}

178. Pelvic elements in adults, at the level of the acetabulum: unfused or partial fusion (0); completely fused (1).

179. Ilium/ischium, distal co-ossification to completely enclose the ilioischiadic fenestra: absent (0); present (1).

180. Preacetabular process of ilium twice as long as postacetabular process: absent (0); 
present (1).

181. Preacetabular ilium: approach on midline, open, or cartilaginous connection (0); co-ossified, dorsal closure of "iliosynsacral canals" (1).

182. Ilium, m. cuppedicus fossa as broad, mediolaterally oriented surface directly cranioventral to acetabulum: present (0); surface absent, insertion variably marked by a small entirely lateral fossa cranial to acetabulum (1).

183. Preacetabular pectineal process (Baumel and Witmer, 1993): absent (0); present as a small flange (1); present as a well-projected flange (2). (ORDERED)

184. Small acetabulum, acetabulum/ilium length ratio equal to or smaller than 0.11 : absent (0); present (1).

185. Prominent antitrochanter: caudally directed (0); caudodorsally directed (1).

186. Postacetabular process shallow, less than $50 \%$ of the depth of the preacetabular wing at the acetabulum: absent (0); present (1).

187. Iliac brevis fossa: present (0); absent (1).

188. Ischium: two-thirds or less the length of the pubis (0); more than two-thirds the length of the pubis (1).

189. Obturator process of ischium: prominent (0); reduced or absent (1). The ischium of Archaeopteryx is forked distally; the thicker cranioventrally oriented fork is here interpreted to be the obturator process.

190. Ischium, caudal demarcation of the obturator foramen: absent (0); present, developed as a small flange or raised scar contacting/fused with pubis and demarcating the obturator foramen distally (1). 
191. Ischium with a proximodorsal (or proximocaudal) process: absent (0); present (1).

192. Ischiadic terminal processes forming a symphysis: present (0); absent (1).

193. Orientation of proximal portion of pubis: cranially to subvertically oriented (0); retroverted, separated from the main synsacral axis by an angle ranging between $65^{\circ}$ and $45^{\circ}$ (1); more or less parallel to the ilium and ischium (2). (ORDERED)

194. Pubic pedicel: cranioventrally projected (0); ventrally or caudoventrally projected (1).

195. Pubic pedicel of ilium very compressed laterally and hook-like: absent (0), present (1).

196. Pubic shaft laterally compressed throughout its length: absent (0); present (1).

197. Pubic apron: one-third or more the length of the pubis (0); shorter (1); absent (absence of symphysis) (2). (ORDERED)

198. Pubic foot: present (0); absent (1). This refers to the distinct long, caudodorsal tapered expansion of the distal pubis, as opposed to the gradual expansion of the distal pubes present in taxa such as Confuciusornis.

\section{Pelvic Limb}

199. Femur with distinct fossa for the capital ligament: absent (0); present (1).

200. Femoral neck: present (0); absent (1).

201. Femoral anterior trochanter: separated from the greater trochanter (0); fused to it, forming a trochanteric crest with a laterally curved edge (1); fused to it, forming a 
trochanteric crest with a flattened edge (2).

202. Femoral trochanteric crest: projects proximally beyond femoral head (0); equal in proximal projection (1); does not project beyond femoral head (2).

203. Femoral posterior trochanter: present, developed as a slightly projected tubercle or flange (0); hypertrophied, "shelf-like" conformation (1) (in combination with development of the trochanteric shelf; see Hutchinson, 2001); absent (2).

204. Femur with prominent patellar groove: absent (0); present as a continuous extension onto the distal shaft (1); present and separated from the shaft by a slight ridge, giving it a pocketed appearance (2).

205. Femur: ectocondylar tubercle and lateral condyle separated by deep notch (0); ectocondylar tubercle and lateral condyle contiguous but without developing a tibiofibular crest (1); tibiofibular crest present, defining laterally a fibular trochlea (2). Proximal to the lateral condyle in theropod dinosaurs there is a caudal projection known as the ectocondylar tubercle. It is hypothesized that this tubercle is homologous to the precursor to the tibiofibular crest, formed through the connection of the ectocondylar tubercle and the lateral condyle. (ORDERED)

206. Caudal projection of the lateral border of the distal end of the femur, proximal and contiguous to the ectocondylar tubercle/tibiofibular crest: absent (0); present (1).

207. Femoral popliteal fossa distally bounded by a complete transverse ridge: absent (0); present (1).

208. Fossa for the femoral origin of $\mathrm{m}$. tibialis cranialis: absent (0); present (1). 
209. Tibia, calcaneum, and astragalus: unfused or poorly co-ossified (sutures still visible) (0); complete fusion of tibia, calcaneum, and astragalus (1).

210. Round proximal articular surface of tibiotarsus: absent (0); present (1).

211. Tibiotarsus, proximal articular surface: flat (0); angled so that the medial margin is elevated with respect to the lateral margin (1).

212. Tibiotarsus, cnemial crests: absent (0); present, one (1); present, two (2).

213. Tibia, caudal extension of articular surface for distal tarsals/tarsometatarsus: absent, articular restricted to distalmost edge of caudal surface (0); well-developed caudal extension, sulcus cartilaginis tibialis of Aves [11], distinct surface extending up the caudal surface of the tibiotarsus (1); with well-developed, caudally projecting medial and lateral crests (2). (ORDERED)

214. Extensor canal on tibiotarsus: absent (0); present as an emarginate groove (1); groove bridged by an ossified supratendinal bridge (2). (ORDERED)

215. Tibia/tarsal-formed condyles: medial condyle projecting farther cranially than lateral condyle (0); equal in cranial projection (1).

216. Tibia/tarsal-formed condyles, mediolateral widths: medial condyle wider (0); approximately equal (1); lateral condyle wider (2). (ORDERED)

217. Tibia/tarsal-formed condyles: gradual sloping of condyles towards midline of tibiotarsus (0); no tapering of either condyle (1).

218. Proximal end of the fibula: prominently excavated by a medial fossa (0); nearly flat (1).

219. Fibula, tubercle for $\mathrm{m}$. iliofibularis: craniolaterally directed (0); laterally directed 
(1); caudolaterally or caudally directed (2). (ORDERED)

220. Fibula, distal end reaching the proximal tarsals: present (0); absent (1).

221. Distal tarsals in adults: free (0); completely fused to the metatarsals (1). Any specimen that is inferred to be a juvenile should be scored as a "?" in order to account for the possibility of ontogenetic change.

222. Metatarsals II-IV, intermetatarsal fusion: absent or minimal co-ossification (0); partial fusion, sutural contacts easily discernible (1); completely or nearly completely fused, sutural contacts absent or poorly demarcated (2). (ORDERED)

223. Proximal end of metatarsus: plane of articular surface perpendicular to longitudinal axis of metatarsus (0); strongly inclined dorsally (1).

224. Metatarsal V: present (0); absent (1).

225. Proximal end of metatarsal III: in the same plane as metatarsals II and IV (0); plantarly displaced with respect to metatarsals II and IV (1).

226. Tarsometatarsal proximal vascular foramen/foramina: absent (0); one between metatarsals III and IV (1); two (2).

227. Metatarsals, relative mediolateral width: metatarsal IV approximately the same width as metatarsals II and III (0); metatarsal IV narrower than metatarsals II and III (1); metatarsal IV greater in width than either metatarsal II or III (2).

228. Well-developed tarsometatarsal intercotylar eminence: absent (0); present, low and rounded (1); present, high and peaked (2).

229. Tarsometatarsus, projected surface and/or grooves on proximocaudal surface (associated with the passage of tendons of the pes flexors in Aves; hypotarsus): 
absent (0); developed as caudal projection with flat caudal surface (1); projection, with distinct crests and grooves (2); at least one groove enclosed by bone caudally (3). (ORDERED)

230. Plantar surface of tarsometatarsus excavated: absent (0); present (1).

231. Tarsometatarsal distal vascular foramen completely enclosed by metatarsals III and IV: absent (0); present (1).

232. Metatarsal I: straight (0); J-shaped, the articulation of the hallux is located on the same plane as the attachment surface of the metatarsal I (1); J-shaped; the articulation of the hallux is perpendicular to the attachment surface (2); the distal half of the metatarsal I is laterally deflected so that the laterodistal surface is concave (3).

233. Metatarsal II tubercle (associated with the insertion of the tendon of the $\mathrm{m}$. tibialis cranialis in Aves): absent (0); present, on approximately the center of the proximodorsal surface of metatarsal II (1); present, developed on lateral surface of metatarsal II, at contact with metatarsal III or on lateral edge of metatarsal III (2).

\section{(ORDERED)}

234. Metatarsal II, distal plantar surface, fossa for metatarsal I (fossa metatarsi I [11]): absent (0); shallow notch (1); conspicuous ovoid fossa (2). (ORDERED)

235. Relative position of metatarsal trochleae: trochlea III more distal than trochleae II and IV (0); trochlea III at same level as trochlea IV, both more distal than trochlea II (1); trochlea III at same level as trochleae II and IV (2); distal extent of trochlea III intermediate to trochlea IV and II where trochlea IV projects furthest 
distally (3).

236. Metatarsal II, distal extent of metatarsal II relative to metatarsal IV: approximately equal in distal extent (0); metatarsal II shorter than metatarsal IV but reaching distally farther than base of metatarsal IV trochlea (1); metatarsal II shorter than metatarsal IV, reaching distally only as far as base of metatarsal IV trochlea (2).

237. Distal tarsometatarsus, trochlea in distal view: aligned in a single plane (0); metatarsal II slightly displaced plantarly with respect to III and IV (1); metatarsal II strongly displaced plantarly in respect to III and IV, such that there is little or no overlap in medial view (2).

238. Trochlea of metatarsal II broader than the trochlea of metatarsal III: absent (0); present (1).

239. Metatarsal III, trochlea in plantar view, proximal extent of lateral and medial edges of trochlea: trochlear edges approximately equal in proximal extent (0); medial edge extends farther (1).

240. Distal end of metatarsal II strongly curved medially: absent (0); present (1).

241. Digit IV phalanges in distal view, medial trochlear rim enlarged with respect to lateral trochlear rim: absent (0); present (1); greatly enlarged with the lateral trochlea reduced to a rounded peg (2).

242. Completely reversed hallux (arch of ungual phalanx of digit I opposing the arch of the unguals of digits II-IV): absent (0); present (1).

243. Size of claw of hallux relative to other pedal claws: shorter, weaker, and smaller 
(0); similar in size (1); longer, more robust, and larger (2).

\section{Integument}

244. Alula: absent (0); present (1).

245. Fan-shaped feathered tail composed of more than two elongate retrices: absent (0); present (1).

\section{Added Characters}

246. Expanded lateral processes of pygostyle: restricted to cranial portion, forming a strong distal constriction (0); extends entire length of pygostyle, forming a slight distal constriction (1); extends caudally past the pygostyle midline, forming a deep incision in the distal margin (2).

247. Tibia/femur length ratio: less than $110 \%$ (0); 110-130\% (1); larger than $130 \%$ (2).

248. Metatarsal I/tarsometatarsus (metatarsal I/metatarsal III if no tarsometatarsus presents) length ratio: larger than 1/3 (0); 1/3-1/4 (1); less than 1/4 (2). 